\title{
CHEVALLEY GROUPS OF POLYNOMIAL RINGS OVER DEDEKIND DOMAINS
}

\author{
A. STAVROVA
}

\begin{abstract}
Let $R$ be a Dedekind domain, and let $G$ be a split reductive group, i.e. a ChevalleyDemazure group scheme, of rank $\geq 2$. We prove that $G\left(R\left[x_{1}, \ldots, x_{n}\right]\right)=G(R) E\left(R\left[x_{1}, \ldots, x_{n}\right]\right)$ for any $n \geq 1$. This extends the corresponding results of A. Suslin and F. Grunewald, J. Mennicke, and L. Vaserstein for $G=\mathrm{SL}_{N}, \mathrm{Sp}_{2 N}$. We also deduce some corollaries of the above result for regular rings $R$ of higher dimension and discrete Hodge algebras over $R$.
\end{abstract}

\section{INTRODUCTION}

A. Suslin $[\mathrm{Su}$, Corollary 6.5] established that for any regular $\operatorname{ring} R$ of dimension $\leq 1$, any $N \geq 3$, and any $n \geq 1$, one has

$$
\mathrm{SL}_{N}\left(R\left[x_{1}, \ldots, x_{n}\right]\right)=\mathrm{SL}_{N}(R) E_{N}\left(R\left[x_{1}, \ldots, x_{n}\right]\right),
$$

where $E_{N}\left(R\left[x_{1}, \ldots, x_{n}\right]\right)$ is the elementary subgroup, i.e. the subgroup generated by elementary matrices $I+t e_{i j}, 1 \leq i \neq j \leq N, t \in R\left[x_{1}, \ldots, x_{n}\right]$. In particular, this implies

$$
\mathrm{SL}_{N}\left(\mathbb{Z}\left[x_{1}, \ldots, x_{n}\right]\right)=E_{N}\left(\mathbb{Z}\left[x_{1}, \ldots, x_{n}\right]\right) .
$$

A later theorem of A. Suslin and V. Kopeiko [SuK, Theorem 7.8] together with the homotopy invariance of orthogonal $K$-theory (see [Kar73, Corollaire 0.8], [Hor05, Corollary 1.12], or [Sch17, Theorem 9.8]) implies a similar result for even orthogonal groups $S_{2 N}, N \geq 3$, under the additional assumption $2 \in R^{\times}$. F. Grunewald, J. Mennicke, and L. Vaserstein GMV91] extended the result of Suslin to symplectic groups $\mathrm{Sp}_{2 N}, N \geq 2$, and a slightly larger class of rings $R$, namely, locally principal ideal rings. One says that a (commutative associative) ring $A$ with 1 is a locally principal ideal ring, if for every maximal ideal $m$ of $A$ the localization $A_{m}$ is a principal ideal ring.

Our aim is to extend the above results to all Chevalley-Demazure group schemes of isotropic rank $\geq 2$. By a Chevalley-Demazure group scheme we mean a split reductive group scheme in the sense of [SGA3]. These group schemes are defined over $\mathbb{Z}$. We say that a ChevalleyDemazure group scheme $G$ has isotropic rank $\geq n$ if and only if every irreducible component of its root system has rank $\geq n$. For any commutative ring $R$ with 1 and any fixed choice of a pinning, or épinglage of $G$ in the sense of [SGA3], we denote by $E$ the elementary subgroup functor of $G$. That is, $E(R)$ is the subgroup of $G(R)$ generated by elementary root unipotent elements $x_{\alpha}(r), \alpha \in \Phi, r \in R$, in the notation of [Ch55, Ma], where $\Phi$ is the root system of $G$. If $G$ has isotropic rank $\geq 2$, then $E$ is independent of the choice of the pinning [PSt].

Our main result is the following theorem. Since $\mathrm{SL}_{2}(\mathbb{Z}[x]) \neq E_{2}(\mathbb{Z}[x])[\mathrm{C}$, it cannot be extended to the case of isotropic rank 1.

The author is a winner of the contest "Young Russian Mathematics". The work was supported by the RFBR grant 18-31-20044. 
Theorem 1.1 (Theorem [3.4). Let $R$ be a locally principal ideal ring, and let $G$ be a simply connected Chevalley-Demazure group scheme of isotropic rank $\geq 2$. Then $G\left(R\left[x_{1}, \ldots, x_{n}\right]\right)=$ $G(R) E\left(R\left[x_{1}, \ldots, x_{n}\right]\right)$ for any $n \geq 1$.

Theorem 1.1 for Dedekind domains was previously claimed by M. Wendt [W], Proposition 4.7], however, his proof was incorrect [Ste13, p. 91]. We give another proof along the lines similar to GMV91. The case where $R$ is a field was done earlier in St14] in a more general context of isotropic reductive groups.

Following [Ma], we say that a Dedekind domain $R$ is of arithmetic type, if $R=O_{S}$ is the ring of $S$-integers of a global field $k$ with respect to a finite non-empty set $S$ of primes containing all archimedean primes.

Corollary 1.2. Let $R$ be a Dedekind domain of arithmetic type (e.g. $R=\mathbb{Z}$ ), and let $G$ be a simply connected Chevalley-Demazure group scheme of isotropic rank $\geq 2$. Then $G\left(R\left[x_{1}, \ldots, x_{n}\right]\right)=E\left(R\left[x_{1}, \ldots, x_{n}\right]\right)$ for any $n \geq 1$.

Proof. This follows from Theorem 1.1 and [Ma, Théorème 12.7], which says that $G(R)=$ $E(R)$.

Note that [Lam06, p. 57] presents an example (due to J. Stallings) of a Dedekind domain $D$ such that $\mathrm{SL}_{3}(D) \neq E_{3}(D)$, hence Corollary 1.2 does not hold for arbitrary Dedekind domains.

A commutative $R$-algebra of the form $A=R\left[x_{1}, \ldots, x_{n}\right] / I$, where $I$ is an ideal generated by monomials, is called a discrete Hodge algebra over $R$. If $I$ is generated by square-free monomials, $A$ is called a square-free discrete Hodge algebra. The simplest example of such an algebra is $R[x, y] / x y$. Square-free discrete Hodge algebras over a field are also called Stanley-Reisner rings.

Corollary 1.3. Let $R$ be a Dedekind domain, and let $G$ be a simply connected ChevalleyDemazure group scheme of isotropic rank $\geq 2$. Then $G(A)=G(R) E(A)$ for any discrete Hodge algebra $A$ over $R$. In particular, if $R$ is of arithmetic type, then $G(A)=E(A)$.

Proof. This follows from [St19, Corollary 1.5] and Corollary 1.2 ,

For non-simply connected Chevalley-Demazure group schemes, such as $\mathrm{SO}_{n}, n \geq 5$, we deduce the following result; see $\S 3$ for the proof.

Corollary 1.4. Let $R$ be a locally principal ideal domain, and let $G$ be any Chevalley-Demazure group scheme of isotropic rank $\geq 2$. Then $G\left(R\left[x_{1}, \ldots, x_{n}\right]\right)=G(R) E\left(R\left[x_{1}, \ldots, x_{n}\right]\right)$ for any $n \geq 1$.

Using a version of Lindel's lemma [L] and Néron-Popescu desingularization [Pop90, one may extend the above results to higher-dimensional regular rings in place of Dedekind domains. For equicharacteristic regular rings this was done earlier in St14. The following theorem is proved in $\S$.

Theorem 1.5. Let $G$ be a Chevalley-Demazure group scheme of isotropic rank $\geq 2$. Let $R$ be a regular ring such that every maximal localization of $R$ is either essentially smooth over a Dedekind domain with perfect residue fields, or an unramified regular local ring. Then $G(R[x])=G(R) E(R[x])$. Moreover, $G(A)=G(R) E(A)$ for any square-free discrete Hodge algebra $A$ over $R$. 
Let us mention a few other ramifications of known results yielded by Theorem 1.1,

Combining Corollary 1.3 with the main result of [EJZK17], one concludes that $G(A)$ has Kazhdan's property (T) for any simply connected Chevalley-Demazure group scheme $G$ of isotropic rank $\geq 2$ and any discrete Hodge algebra $A$ over $\mathbb{Z}$; in particular, $G\left(\mathbb{Z}\left[x_{1}, \ldots x_{n}\right]\right)$ has Kazhdan's property (T).

Combining Corollary 1.3 with the main result of [RR], one concludes that the congruence kernel of $G(A)$ is central in $G(A)$ for any simply connected Chevalley-Demazure group scheme $G$ of isotropic rank $\geq 2$ and any discrete Hodge algebra $A$ over $R$, where $R$ is a Dedekind domain of arithmetic type, satisfying $2 \in R^{\times}$if the root system of $G$ has components of type $C_{n}$ or $G_{2}$.

\section{A LOCAL-GLOBAL PRINCIPLE}

Throughout this section, $R$ is any commutative ring with $1, G$ is a Chevalley-Demazure group scheme of isotropic rank $\geq 2$, and $E$ denotes its elementary subgroup functor.

For any $s \in R$ we denote by $R_{s}$ the localization of $R$ at $s$, and by $F_{s}: R \rightarrow R_{s}$ the localization homomorphism, as well as the induced homomorphism $G(R) \rightarrow G\left(R_{s}\right)$. Similarly, for any maximal ideal $m$ of $R$ we denote by $F_{m}: R \rightarrow R_{m}$ the localization homomorphism, as well as the induced homomorphism $G(R) \rightarrow G\left(R_{m}\right)$.

We will need the following generalization of the Quillen-Suslin local-global principle for polynomial rings in one variable (see [Su, Theorem 3.1], [SuK, Corollary 4.4], [PSt, Lemma 17], [Ste13, Theorem 5.4]) to the case of several variables.

Lemma 2.1. Let $R$ be any commutative ring. Fix $n \geq 1$. If $g \in G\left(R\left[x_{1}, \ldots, x_{n}\right]\right)$ satisfies $F_{m}(g) \in E\left(R_{m}\left[x_{1}, \ldots, x_{n}\right]\right)$ for any maximal ideal $m$ of $R$, then $g \in G(R) E\left(R\left[x_{1}, \ldots, x_{n}\right]\right)$.

The proof of Lemma 2.1 uses the following three standard lemmas whose idea goes back to Q, Lemma 1].

Lemma 2.2. Let $H$ be any affine $R$-scheme of finite type. Fix $0 \neq s \in R$, and let $F_{s}$ : $H(R[z]) \rightarrow H\left(R_{s}[z]\right)$ be the localization map. For any $g(z), h(z) \in H(R[z])$ such that $h(0)=$ $g(0)$ and $F_{s}(g(z))=F_{s}(h(z))$ there is $n \geq 0$ such that $g\left(s^{n} z\right)=h\left(s^{n} z\right)$.

Proof. Since $H$ is an affine $R$-scheme of finite type, there is a closed embedding $H \rightarrow \mathbb{A}_{R}^{k}$ for some $k \geq 0$. Hence it is enough to prove the claim for $H=\mathbb{A}_{R}^{k}$. If $k=0$, then $g(z)=g(0)=$ $h(0)=h(z)$. If $k \geq 1$, the claim readily reduces to the case $k=1$, that is, $g(z), h(z) \in R[z]$. Since $F_{s}(g(z))=F_{s}(h(z))$, there is $n \geq 0$ such that $s^{n} g(z)=s^{n} h(z)$. Since $g(0)=h(0)$, this implies $g\left(s^{n} z\right)=h\left(s^{n} z\right)$.

Lemma 2.3. [Ste13, Theorem 5.2] Fix $s \in R$, and let $F_{s}: G(R[z]) \rightarrow G\left(R_{s}[z]\right)$ be the localization homomorphism. For any $g(z) \in E\left(R_{s}[z], z R_{s}[z]\right)$ there exist $h(z) \in E(R[z], z R[z])$ and $k \geq 0$ such that $F_{s}(h(z))=g\left(s^{k} z\right)$.

Proof. The statement is a particular case of [Ste13, Theorem 5.2] if the root system $\Phi$ of $G$ is irreducible. Assume that $\Phi$ has several irreducible components $\Phi_{i}$. By [SGA3, Exp. XXVI Prop. 6.1] $G$ contains semisimple Chevalley-Demazure subgroup schemes $G_{i}$ of type $\Phi_{i}$ whose elementary subgroup functors $E_{i}$ are generated by elementary root unipotents corresponding to roots in $\Phi_{i}$. Chevalley commutator relations imply that $E$ is a direct product of all $E_{i}$. This reduces the claim to the case where $\Phi$ is irreducible. 
Lemma 2.4. For any $g(x) \in G(R[x])$ such that $F_{s}(g(x))$ lies in $E\left(R_{s}[x]\right)$, there exists $k \geq 0$ such that $g(a x) g(b x)^{-1} \in E(R[x])$ for all $a, b \in R$ satisfying $a \equiv b \bmod s^{k}$.

Proof. Consider the element $f(z)=g(x(y+z)) g(x y)^{-1} \in G(R[x, y, z])$. Observe that $F_{s}(f(z)) \in$ $E\left(R_{s}[x, y, z]\right)$ and $f(0)=1$. Since $F_{s}(g(x)) \in E\left(R_{s}[x]\right)$ and $f(0)=1$, we have $F_{s}(f(z)) \in$ $E\left(R_{s}[x, y, z], z R_{s}[x, y, z]\right)$ (e.g. by [St14, Lemma 4.1]). Now by Lemma 2.3 there exist $h(z) \in$ $E(R[x, y, z], z R[x, y, z])$ and $k \geq 1$ such that $F_{s}(h(z))=F_{s}\left(f\left(s^{k} z\right)\right)$. By Lemma 2.2 there is $l \geq 1$ such that $h\left(s^{l} z\right)=f\left(s^{l+k} z\right)$. Then $g\left(x\left(y+s^{l+k} z\right)\right) g(x y)^{-1}$ lies in $\mathrm{E}_{P}(R[x, y, z])$. It remains to set $y=b$ and to choose a suitable $z$ depending on $a$.

Proof of Lemma 2.1. For any maximal ideal $m$ of $R$, since $F_{m}(g) \in E\left(R_{m}\left[x_{1}, \ldots, x_{n}\right]\right)$, there is $s \in R \backslash m$ such that $F_{s}(g) \in E\left(R_{s}\left[x_{1}, \ldots, x_{n}\right]\right)$. Choose a finite set of elements $s=s_{i} \in R \backslash m_{i}$, $1 \leq i \leq N$ as above, so that $1=\sum_{i=1}^{N} c_{i} s_{i}$ for some $c_{i} \in R$. Consider $g$ as a function $g\left(x_{1}\right)$ of $x_{1}$. By Lemma 2.4 there are $k_{i} \geq 1$ such that $g\left(a x_{1}\right) g\left(b x_{1}\right)^{-1} \in E\left(R\left[x_{1}, \ldots, x_{n}\right]\right)$ for any $a, b \in R\left[x_{2}, \ldots, x_{n}\right]$ satisfying $a \equiv b\left(\bmod s_{i}^{k_{i}}\right)$. Since $s_{i}$ generate the unit ideal, their powers $s_{i}^{k_{i}}$ also generate the unit ideal, and we can replace $s_{i}$ by these powers without loss of generality. Set $a_{j}=\sum_{i=1}^{N-j} c_{i} s_{i}, 0 \leq j \leq N$. Then $a_{j+1} \equiv a_{j}\left(\bmod s_{n-j}\right)$, and

$$
g\left(x_{1}\right)=\left(\prod_{j=0}^{N-1} g\left(a_{j} x_{1}\right) g\left(a_{j+1} x_{1}\right)\right)^{-1} g(0) .
$$

Then $g\left(x_{1}\right) \in E\left(R\left[x_{1}, \ldots, x_{n}\right]\right) g(0)$. Since $g(0) \in G\left(R\left[x_{2}, \ldots, x_{n}\right]\right)$, we can proceed by induction.

Lemma 2.5. Fix $n \geq 1$. One has $G\left(R\left[x_{1}, \ldots, x_{n}\right]\right)=G(R) E\left(R\left[x_{1}, \ldots, x_{n}\right]\right)$ if and only if $G\left(R_{m}\left[x_{1}, \ldots, x_{n}\right]\right)=G\left(R_{m}\right) E\left(R_{m}\left[x_{1}, \ldots, x_{n}\right]\right)$ for every maximal ideal $m$ of $R$.

Proof. For the direct implication, see [St14, Lemma 4.2]. To prove the converse, it is enough to show that $g\left(x_{1}, \ldots, x_{n}\right) \in G\left(R\left[x_{1}, \ldots, x_{n}\right]\right)$ such that $g(0, \ldots, 0)=1$ satisfies $g\left(x_{1}, \ldots, x_{n}\right) \in$ $E\left(R\left[x_{1}, \ldots, x_{n}\right]\right)$. For every maximal ideal $m$ of $R$, by assumption, one has $F_{m}\left(g\left(x_{1}, \ldots, x_{n}\right) \in\right.$ $G\left(R_{m}\right) E\left(R_{m}\left[x_{1}, \ldots, x_{n}\right]\right)$, and $g(0, \ldots, 0)=1$ implies $F_{m}\left(g\left(x_{1}, \ldots, x_{n}\right)\right) \in E\left(R_{m}\left[x_{1}, \ldots, x_{n}\right]\right)$. Then Lemma 2.1 finishes the proof.

\section{Proof of THE MAIN THEOREM}

The following result follows from stability results for non-stable $K_{1}$-funtors of Chevalley groups [Ste78, Plo93].

Lemma 3.1. Let $R$ be a Noetherian ring of Krull dimension $\leq 1$. If $\mathrm{SL}_{2}(R)=E_{2}(R)$, then $G(R)=E(R)$ for any simply connected Chevalley-Demazure group scheme $G$ over $R$.

Proof. By [Bas68, p. 102] the maximal ideal spectrum of $R$ is a Noetherian topological space of dimension $\leq 1$. By [Ste78, Theorem 1.4] this implies that $R$ satisfies the absolute stable range condition $A S R_{3}$, and hence also Bass' stable range condition $S R_{3}$ in the sense of [Ste78, p. 86]. Then by [Ste78, Theorem 2.2] (see also [Ste78, Corollary 2.3]) suitable inclusions of $\mathrm{SL}_{2}$ into $G$ induce surjections $\mathrm{SL}_{2}(R) / E_{2}(R) \rightarrow G(R) / E(R)$ for every simply connected ChevalleyDemazure group scheme $G$ corresponding to an irreducible root system of classical type $A_{n}$, $n \geq 1, C_{n}, n \geq 2, D_{n}, n \geq 3$, or $B_{n}, n \geq 2$. By [Ste78, Theorem 4.1] and [Plo93, Corollary 3] the same also holds for $G$ of type $G_{2}, F_{4}, E_{6}, E_{7}$, and $E_{8}$. Consequently, $G(R)=E(R)$ for any simply connected Chevalley-Demazure group scheme $G$ over $R$. 
For any commutative ring $R$ with 1 , denote by $R(x)$ the localization of $R[x]$ at the set of all monic polynomials.

Lemma 3.2. Let $R$ be a discrete valuation ring or a local Artinian ring. Then $G(R(x))=$ $E(R(x))$ for any simply connected Chevalley-Demazure group scheme $G$ over $R$.

Proof. Since $R$ is a commutative Noetherian ring, by [Lam06, Ch. IV, Proposition 1.2] $R(x)$ has the same Krull dimension as $R$. If $R$ is Artinian, then $R(x)$ is also Artinian, and hence a finite product of local rings. Then $S L_{n}(R(x))=E_{n}(R(x))$ for all $n \geq 2$. If $R$ is a discrete valuation ring, then also $S L_{n}(R(x))=E_{n}(R(x))$ for all $n \geq 2$ by Lam06, Ch. IV, Corollary 6.3] (a corollary of [Mur66, Proposition 1']). Hence by Lemma 3.1 one has $G(R(x))=E(R(x))$ in both cases.

We will also use the following lemma, that was established in [Su, Corollary 5.7] for $G=\mathrm{GL}_{n}$.

Lemma 3.3. [St15, Lemma 2.7] Let $A$ be a commutative ring, and let $G$ be a reductive group scheme over $A$, such that every semisimple normal subgroup of $G$ is isotropic. Assume moreover that for any maximal ideal $m \subseteq A$, every semisimple normal subgroup of $G_{A_{m}}$ contains $\left(\mathbf{G}_{\mathrm{m}, A_{m}}\right)^{2}$. Then for any monic polynomial $f \in A[x]$ the natural homomorphism

$$
G(A[x]) / E(A[x]) \rightarrow G\left(A[x]_{f}\right) / E\left(A[x]_{f}\right)
$$

is injective.

Now we are ready to establish the main theorem for simply connected semisimple ChevalleyDemazure group schemes.

Theorem 3.4. Let $R$ be a locally principal ideal ring or Artinian ring. Then

$$
G\left(R\left[x_{1}, \ldots, x_{n}\right]\right)=G(R) E\left(R\left[x_{1}, \ldots, x_{n}\right]\right)
$$

for any simply connected Chevalley-Demazure group scheme $G$ over $R$ of isotropic rank $\geq 2$ and any $n \geq 1$.

Proof. For every maximal ideal $m$ of $R$, the $\operatorname{ring} R_{m}$ is a local principal ideal domain, i.e. a discrete valuation ring, or a local Artinian ring, In both cases $R_{m}$ is a local Noetherian ring of Krull dimension $\leq 1$. By [Lam06, Ch. IV, Proposition 1.2] $R_{m}\left(x_{1}\right)$ has the same Krull dimension as $R_{m}$. If $R_{m}$ is Artinian, then $R_{m}\left(x_{1}\right)$ is also Artinian. If $R_{m}$ is a discrete valuation ring, then $R_{m}\left(x_{1}\right)$ is a principal ideal domain by [Lam06, Ch. IV, Corollary 1.3]. Hence by induction hypothesis

$$
G\left(R_{m}\left(x_{1}\right)\left[x_{2}, \ldots, x_{n}\right]\right)=G\left(R_{m}\left(x_{1}\right)\right) E\left(R_{m}\left(x_{1}\right)\left[x_{2}, \ldots, x_{n}\right]\right) .
$$

Then by Lemma $3.2 G\left(R_{m}\left(x_{1}\right)\left[x_{2}, \ldots, x_{n}\right]\right)=E\left(R_{m}\left(x_{1}\right)\left[x_{2}, \ldots, x_{n}\right]\right)$. Then by Lemma 3.3 we have $G\left(R_{m}\left[x_{1}, \ldots, x_{n}\right]\right)=E\left(R_{m}\left[x_{1}, \ldots, x_{n}\right]\right)$. Then Lemma 2.5 finishes the proof.

To pass from simply connected Chevalley-Demazure group schemes to general ones, we use the following reduction lemma.

Lemma 3.5. Let $G$ be a Chevalley-Demazure group scheme, and let $E$ be an elementary subgroup functor of $G$. Let $G^{\mathrm{sc}}$ be the simply connected cover of the adjoint semisimple group scheme $G^{\mathrm{ad}}=G / \operatorname{Cent}(G)$, and let $E^{\mathrm{sc}}$ be its elementary subgroup functor corresponding to the pinning compatible with that of $G$. Let $A$ be a normal Noetherian integral domain. If one has $G^{\mathrm{sc}}(A[x])=G^{\mathrm{sc}}(A) E^{\mathrm{sc}}(A[x])$, then $G(A[x])=G(A) E(A[x])$. 
Proof. There is a short exact sequence of $\mathbb{Z}$-group schemes

$$
1 \rightarrow[G, G] \rightarrow G \rightarrow T \rightarrow 1
$$

for a split $\mathbb{Z}$-torus $T$. Here the group $[G, G]$ is the algebraic derived subgroup scheme of $G$ in the sense of [SGA3, Exp. XXII, §6.2]. It is a semisimple Chevalley-Demazure group scheme, and $E(A) \leq[G, G](A)$. Since $T(A[x])=T(A)$, the exact sequence

$$
1 \rightarrow[G, G](A[x]) \rightarrow G(A[x]) \rightarrow T(A[x])
$$

implies that it is enough to prove the claim for $[G, G]$. In other words, we may assume that $G$ is semisimple. Then there is a short exact sequence of algebraic groups

$$
1 \rightarrow C \stackrel{i}{\rightarrow} G^{\mathrm{sc}} \stackrel{\pi}{\rightarrow} G \rightarrow 1
$$

where $C$ is a group of multiplicative type over $\mathbb{Z}$, central in $G^{\mathrm{sc}}$. Write the respective "long" exact sequences over $A[x]$ and $A$ with respect to fppf topology. Adding the maps induced by the homomorphism $\rho: A[x] \rightarrow A, x \mapsto 0$, we obtain a commutative diagram

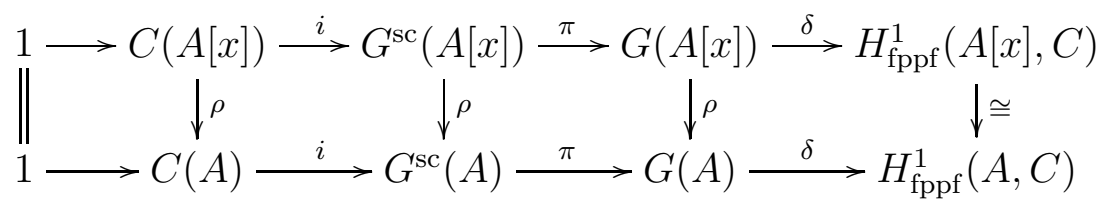

Here the rightmost vertical arrow is an isomorphism by [CTS, Lemma 2.4]. Take any $g \in$ $\operatorname{ker}(\rho: G(A[x]) \rightarrow G(A))$. It is enough to show that $g \in E(A[x])$.

We have $\delta(g)=1$, hence there is $\tilde{g} \in G^{\mathrm{sc}}(A[x])$ with $\pi(\tilde{g})=g$. Clearly, $\rho(\tilde{g}) \in C(A)$, and hence

$$
\tilde{g} \in C(A) \cdot \operatorname{ker}\left(\rho: G^{\mathrm{sc}}(A[x]) \rightarrow G^{\mathrm{sc}}(A)\right) \subseteq C(A) E^{\mathrm{sc}}(A[x]) .
$$

Since $\pi\left(E^{\mathrm{sc}}(A[x])\right)=E(A[x])$, this proves the claim.

Proof of Corollary 1.4. By Lemma 2.5 it is enough to prove the claim for $R_{m}$, where $m$ is any maximal ideal of $R$. Since $R_{m}$ is a discrete valuation ring, the claim follows from Lemma 3.5 and Theorem 1.1 .

\section{EXTENSION TO HIGHER DIMENSIONAL REGULAR RINGS}

In this section we discuss extensions of Theorem 1.1 to rings of polynomials over higher dimensional regular rings $R$. Note that the following result is contained in [St14].

Theorem 4.1. Let $G$ be a Chevalley-Demazure group scheme of isotropic rank $\geq 2$. Let $R$ be an equicharacteristic regular domain. Then $G\left(R\left[x_{1}, \ldots, x_{n}\right]\right)=G(R) E\left(R\left[x_{1}, \ldots, x_{n}\right]\right)$ for any $n \geq 1$.

Proof. The claim follows from [St14, Theorem 1.3], since $R\left[x_{1}, \ldots, x_{n}\right]$ is a regular domain containig a perfect field, for any $n \geq 1$.

Thus, it remains to consider the case of regular domains $R$ of unequal characteristic. Following [W], we rely on the following generalization of Lindel's lemma [L]. See also Pop89, Proposition 2.1] for a slightly weaker version. 
Lemma 4.2. [Dut00, Theorem 1.3] Let $(A, m)$ be a regular local ring of dimension $d+1$, essentially of finite type and smooth over an excellent discrete valuation ring $(V,(\pi))$ such that $K=A / m$ is separably generated over $V / \pi V$. Let $0 \neq a \in m^{2}$ be such that $a \notin \pi A$. Then there exists a regular local subring $(B, n)$ of $(A, m)$, with $B / n=A / m=K$, and such that

(1) $B$ is a localization of a polynomial ring $W\left[x_{1}, \ldots, x_{d}\right]$ at a maximal ideal of the type $\left(\pi, f\left(x_{1}\right), x_{2}, \ldots, x_{d}\right)$ where $f$ is a monic irreducible polynomial in $W\left[x_{1}\right]$ and $(W,(\pi))$ is an excellent discrete valuation ring contained in $A$; moreover $A$ is an étale neighborhood of $B$.

(2) There exists an element $h \in B \cap a A$ such that $B / h B \cong A / a A$ is an isomorphism. Furthermore $h A=a A$.

Lemma 4.3. Let $G$ be a Chevalley-Demazure group scheme $G$ of isotropic rank $\geq 2$. Let $R$ be a Dedekind domain with perfect residue fields. Let $A$ be a regular $R$-algebra that is essentially smooth over $R$. Then $G(A[x])=G(A) E(A[x])$.

Proof. By Lemma 2.5 we can assume that $A$ is local. Then, in particular, $R$ is a regular domain, and hence we can assume that $G$ is simply connected by Lemma 3.5. The map $R \rightarrow A$ factors through a localization $R_{q}$, for a prime ideal $q$ of $R$. If $R_{q}$ is equicharacteristic, we are done by Theorem 4.1. Otherwise $R_{q}=V$ is a discrete valuation ring of characteristic 0 , and hence excellent by Gro65, Scholie 7.8.3]. The residue field of $A$ is a finitely generated field extension of the perfect field $R_{q} / q R_{q}=V / \pi$, hence it is separably generated. Thus, all conditions of Lemma 4.2 are fulfilled.

The rest of the proof proceeds as the proof of [St14, Lemma 6.3], using Lemma 4.2 instead of Lindel's lemma, and Theorem 3.4 instead of [St14, Theorem 1.2]. Namely, one proceeds by induction on $\operatorname{dim} A=d+1$. If $\operatorname{dim} A=1$, we are in the setting of Theorem 3.4. Assume $\operatorname{dim} A \geq 2$. Then $m^{2} \backslash \pi A$ is non-empty, since $A / \pi A$ is an essentially smooth, hence regular, local ring over $V / \pi$, hence a domain. For any $a \in m^{2} \backslash \pi A$, let $B$ and $h \in B \cap a A$ be as in Lemma 4.2. Since $B$ is a localization of a polynomial ring over a discrete valuation ring, which is subject to Theorem 3.4, by Lemma 2.5 one has $G(B[x])=G(B) E(B[x])$. We need to show that any element $g(x) \in G(A[x])$ belongs to $G(A) E(A[x])$. Since $\operatorname{dim} A_{h}<\operatorname{dim} A$, the element $g(x)$ belongs to $G\left(A_{h}\right) E\left(A_{h}[x]\right)$. Clearly, we can assume from the start that $g(0)=1$, then in fact $g(x) \in E\left(A_{h}[x]\right)$. By Lemma $4.2 h$ satisfies $A h+B=A, A h \cap B=B h$. Hence by [St14, Lemma 3.4 (i)] we have $g(x)=g_{1}(x) g_{2}(x)$ for some $g_{1}(x) \in E(A[x])$ and $g_{2}(x) \in G\left(B_{h}[x]\right)$. Then $g_{2}(x) \in G\left(B_{h}[x]\right) \cap G(A[x])=G(B[x])$. Then we have $g_{2}(x) \in G(B) E(B[x])$. Therefore, $g(x) \in G(A) E(A[x])$.

Lemma 4.4. Let $G$ be a Chevalley-Demazure group scheme of isotropic rank $\geq 2$. Let $R$ be a regular ring such that every maximal localization of $R$ is an unramified regular local ring. Then $G(R[x])=G(R) E(R[x])$.

Proof. By Lemma 2.5 we can assume that $R$ is an unramified regular local ring with maximal ideal $m$. If $R$ is equicharacteristic, we are done by Theorem 4.1. If $R$ has characteristic 0 and residual characteristic $p$, then by assumption $p \notin m^{2}$. Then $R$ is geometrically regular over $\mathbb{Z}_{(p)}[\mathrm{Sw}$, p. 4], and hence a filtered inductive limit of regular local rings which are essentially smooth over $\mathbb{Z}_{(p)}$ by [Sw, Corollary 1.3]. Then Lemma 4.3 finishes the proof.

Proof of Theorem 1.5. For the first claim, combine Lemma 2.5, Lemma 4.3, and Lemma 4.4. For the second claim, add [St19, Theorem 1.3]. 


\section{REFERENCES}

[Bas68] H. Bass, Algebraic K-theory, W. A. Benjamin, Inc., New York-Amsterdam, 1968.

[Ch55] C. Chevalley, Sur certains groupes simples, Tohoku Math. J. 7 (1955), 14-66.

[C] P.M. Cohn, On the structure of $G L_{2}$ of a ring, Publ. Math. I.H.É.S. 30 (1966), 365-413.

[CTS] J.-L. Colliot-Thélène, J.-J. Sansuc, Principal homogeneous spaces under flasque tori: applications, Journal of Algebra 106 (1987), 148-205.

[SGA3] M. Demazure, A. Grothendieck, Schémas en groupes, Lecture Notes in Mathematics, vol. 151-153, Springer-Verlag, Berlin-Heidelberg-New York, 1970.

[Dut00] S. P. Dutta, A theorem on smoothness-Bass-Quillen, Chow groups and intersection multiplicity of Serre, Trans. Amer. Math. Soc. 352 (2000), no. 4, 1635-1645.

[EJZK17] M. Ershov, A. Jaikin-Zapirain, M. Kassabov, Property (T) for groups graded by root systems, Mem. Amer. Math. Soc. 249 (2017), no. 1186, v +135.

[Gro65] A. Grothendieck, Éléments de géométrie algébrique. IV. étude locale des schémas et des morphismes de schémas. II, Inst. Hautes Études Sci. Publ. Math. (1965), no. 24, 231.

[GMV91] F. Grunewald, J. Mennicke, L. Vaserstein, On symplectic groups over polynomial rings, Math. Z. 206 (1991), no. 1, 35-56.

[Hor05] J. Hornbostel, $A^{1}$-representability of Hermitian K-theory and Witt groups, Topology 44 (2005), no. 3, 661-687.

[Kar73] M. Karoubi, Périodicité de la K-théorie hermitienne, 301-411. Lecture Notes in Math., Vol. 343.

[Lam06] T. Y. Lam, Serre's problem on projective modules, Springer Monographs in Mathematics, SpringerVerlag, Berlin, 2006.

[L] H. Lindel, On the Bass-Quillen conjecture concerning projective modules over polynomial rings, Invent. Math. 65 (1981), 319-323.

[Ma] H. Matsumoto, Sur les sous-groupes arithmétiques des groupes semi-simples déployés, Ann. Sci. de l'É.N.S. $4^{e}$ série, tome 2, n. 1 (1969), 1-62.

[Mur66] M. Pavaman Murthy, Projective A[X]-modules, J. London Math. Soc. 41 (1966), 453-456.

[PSt] V. Petrov, A. Stavrova, Elementary subgroups of isotropic reductive groups, St. Petersburg Math. J. 20 (2009), 625-644.

[Plo93] E. B. Plotkin, Surjective stabilization of the $K_{1}$-functor for some exceptional Chevalley groups, Journal of Soviet Mathematics 64 (1993), no. 1, 751-766.

[Pop89] D. Popescu, Polynomial rings and their projective modules, Nagoya Math. J. 113 (1989), 121-128.

[Pop90] D. Popescu, Letter to the Editor: General Néron desingularization and approximation, Nagoya Math. J. 118 (1990), 45-53.

[Q] D. Quillen, Projective modules over polynomial rings, Invent. Math. 36 (1976), 167-171.

[RR] A. S. Rapinchuk, I. A. Rapinchuk, Centrality of the congruence kernel for elementary subgroups of Chevalley groups of rank $>1$ over Noetherian rings, Proc. Amer. Math. Soc. 139 (2011), 3099-3113.

[Sch17] M. Schlichting, Hermitian K-theory, derived equivalences and Karoubi's fundamental theorem, J. Pure Appl. Algebra 221 (2017), no. 7, 1729-1844.

[St14] A. Stavrova, Homotopy invariance of non-stable K ${ }_{1}$-functors, J. K-Theory 13 (2014), 199-248.

[St15] A. Stavrova, Non-stable $K_{1}$-functors of multiloop groups, Canad. J. Math. 68 (2016), 150-178.

[St19] A. Stavrova, Isotropic reductive groups over discrete Hodge algebras, J. Homotopy Relat. Str. 14 (2019), 509-524.

[Ste78] M. R. Stein, Stability theorems for $K_{1}, K_{2}$ and related functors modeled on Chevalley groups, Japan. J. Math. (N.S.) 4 (1978), no. 1, 77-108.

[Ste13] A. Stepanov, Elementary calculus in Chevalley groups over rings, J. Prime Res. Math. 9 (2013), 79-95.

[Su] A. A. Suslin, On the structure of the special linear group over polynomial rings. Math. USSR Izv. 11 (1977), 221-238.

[SuK] A.A. Suslin, V.I. Kopeiko, Quadratic modules and the orthogonal group over polynomial rings, J. of Soviet Math. 20 (1982), 2665-2691.

[Sw] R. G. Swan, Néron-Popescu desingularization, in Algebra and Geometry (Taipei, 1995), Lect. Alg. Geom. 2 (1998), 135-198. Int. Press, Cambridge, MA. 
[W] M. Wendt, $\mathbb{A}^{1}$-homotopy of Chevalley groups, J. K-Theory 5 (2010), 245-287.

Chebyshev Laboratory, St. Petersburg State University, 14th Line V.O. 29B, 199178 Saint Petersburg, Russia

E-mail address: anastasia.stavrova@gmail.com 